\title{
CYTOLOGICAL CHARACTERISTICS OF POSTOPERATIVE METASTASES OF PAPILLARY THYROID CANCER DURING THE DEVELOPMENT OF SECONDARY RADIOIODINE REFRACTORINESS
}

\author{
Anna Zelinskaya ${ }^{1}$ \\ ann.zelinskaya@ukr.net \\ Andrey Kvachenyuk ${ }^{1}$ \\ Galina Kulinichenko ${ }^{1}$ \\ Victoria Moroz \\ Taras Shevchenko National University of Kyiv \\ 69 Vyshhorodska str., Kyiv, Ukraine, 04114
}
${ }^{1}$ State Institution "V. P. Komisarenko Institute of Endocrinology and Metabolism of the National Academy of Medical Sciences of Ukraine”
69 Vyshhorodska str., Kyiv, Ukraine, 04114

\begin{abstract}
Radioiodine refractoriness is the main problem in the diagnosis and treatment of papillary thyroid carcinoma. The aim of the study was to investigate the cytological and immunocytochemical changes of thyrocytes in fine-needle aspiration smears of thyroid papillary cancer metastases in the course of the development of secondary radioiodine resistance. A total of 70 postoperative metastases of thyroid papillary cancer (secondary radioiodine refractory metastases, previously responsive to radioiodine, that eventually loses the ability to radioiodine accumulation, radioiodine-avid metastases, primary radioiodine-refractory metastases), immunohistochemical staining of thyroid peroxidase, thyroglobulin, cytokeratin 17 and cytological analysis were performed. Revealing the presence of specific cellular phenotypes and structures in punctuates, a low percentage of thyroid peroxidase and thyroglobulin-positive thyrocytes allows the development of the method of cytological prediction of the radioiodine therapy effectiveness.

Keywords: secondary radioiodine refractory metastases, papillary thyroid carcinoma, fine needle aspiration smears, thyroid peroxidase, thyroglobulin.
\end{abstract}

DOI: $10.21303 / 2504-5695.2020 .001117$

\section{Introduction}

As a result of the Chernobyl accident, there was a sharp increase in incidence of the thyroid cancer [1, 2]. For 20 years of observation, most cases of differentiated thyroid cancer (DTC) have shown the excellent prognosis for more than $95 \%$ of patients thanks to thyroidectomy and radioiodine (RI) - therapy, based on the unique ability of the thyroid cells to accumulate RI [3, 4]. Despite the mainly auspicious prognosis of papillary thyroid carcinoma (PTC) without relapses in most cases even using the standard therapy (thyroidectomy, RI and suppressive therapy), metastases occur during the postoperative period in 2-29\% of cases [3].

Metastases which occur during the postoperative period can be of two types: radioiodine-avid (RIAM) (able to accumulate RI) and radioiodine refractory metastases (RIRM). RIRM develop in $5-20 \%$ of cases, their cells lose their ability to RI accumulation, and RI therapy becomes ineffective for them $[5,6]$. A combination of studies appears to support the notion that survival for patients with RI-refractory DTC distant metastases is around 2.5-3.5 years [7, 8]. Early prediction of RI ability of metastatic DTC can be based on the cytological feature determination of thyrocytes in fine needle aspiration (FNA) smears of the RIRM. There are some ideas about the morphological, immunohistochemical and molecular characteristics of RIRM $[9,10]$. However, the cytological 
studies of FNA smears in RIRM have not yet been conducted and accordingly no data have been obtained on the cytological characteristics of RIRM.

The data regarding RI-refractoriness, classifies it as primary (the metastatic tissue does not ever concentrate RI), secondary (the metastatic tissue loses the ability to concentrate RI after previous evidence of RI-avid disease), and stable metastatic disease [4]. The study of changes of metastases' thyrocytes' characteristics, correlated with the loss of RI accumulation, presents scientific and practical interest. The results can be the basis for developing new methods for predicting and diagnosing radioiodine refractoriness. A suitable model for investigation of changes may be secondary radioiodine-refractory metastases (SRIRM).

The study of antigens' expression - thyroid peroxidase (TPO) and thyroglobulin (Tg) which are involved in the process of RI accumulation and its retention with thyrocytes is of particular interest. We have previously shown that cytokeratin No. 17 (Ck17) is a cytological marker of the preoperative prognosis of RI resistance when determining its expression in FNA smears of primary PTC [11]. The thyrocytes that contains Ck17 are characterized by the weak expression of $\mathrm{Tg}$, and TPO, which also makes it relevant to study the expression of Ck17 antigen in FNA smears of postoperative PTC metastases.

The actual purpose of this work is to carry out cytological and immunocytochemical researches of the thyrocyte population in FNA smears of PTC metastases that appear in the postoperative period, and also to identify cytological changes that occur with the development of secondary radioiodine refractoriness in metastases. There is a unique opportunity to conduct a comparative cytological investigation of SRIRM on different stages of its existence (when metastases accumulate RI (STAGE_1) and when they lose this ability (STAGE_2)).

\section{Materials and methods of research}

\section{1. Patients}

Investigated groups included FNA smears of 70 PTC regional metastases in the neck which were found during the sonographic and scintigraphic examination after thyroidectomy and RI therapy, i. e. in the postoperative period. The specimens were obtained from patients who were examined, diagnosed with the tumor and underwent to thyroid surgery and RI therapy in the Institute of Endocrinology and Metabolism (IEM). PTC metastases were identified by the neck sonography after treating patients with $100 \mathrm{mCi}$ RI. All histopathological diagnoses were established according to the World Health Organisation (WHO) classification at the Laboratory of Morphology of Endocrine System of the IEM [12]. 4 patients had the histological diagnosis - folliculal variant of PTC (FVPC) in the group of RIAM. 3 patients had the histological diagnosis - diffuse-sclerosing variant of PTC (1-in group of RIAM, 1-in group of PRIRM, 1-in group of SRIRM). All other patients had the histological diagnosis - classical variant of PTC. The patients were both women (55) and man (15), with their age ranging from 27 to 61 years (average age 39.29 \pm 17.13 ). This study has been approved by the Ethics Committee of IEM (No. 28/1-KE of 16 June, 2014) and all patients have signed the written form consent. All procedures in this study strictly complied with the guidelines and principles of the Declaration of Helsinki, the Council of the Convention Europe on human rights and biomedicine, as well as relevant provisions of the WHO and the laws of Ukraine.

We have analyzed FNA smears of 15 metastases, previously responsive to RI, that eventually loses the ability to RI accumulation (SRIRM), 25 metastases that were successfully treated, using RI therapy (RIAM) and 30 primary RI-refractory PTC metastases, which does not ever concentrate RI (PRIRM). The specimens were obtained from PTC regional metastases in the neck (paratraheal, jugular (cranial, medial, caudal), submandibular, supraclavicular metastatic lymph node), size from 4 to $33 \mathrm{~mm}$. FNA of thyroid and metastases was performed under ultrasound guidance using a 21-gauge needle. Dried FNA smears were fixed for 5 minutes in methanol, followed by staining in Giemsa dye diluted in $0.067 \mathrm{M}$ phosphate buffer ( $\mathrm{pH}$ 6.4) for 30 minutes.

\section{2. Immunohistochemical staining}

Immunocytochemistry (ICH) was performed on representative Giemsa-stained smears after they have been analyzed cytomorphologically which helps to unmask antigens [13]. We ap- 
plied indirect immunoperoxidase technique to identify certain antigens, using primary monoclonal mouse antibodies against Tg (DAK-Tg6, Dako, Denmark, 1:100 dilution,), TPO (TPO-47, DakoCytomation, Denmark, 1:50 dilution), epithelial cell adhesion molecule (epCAM) (Ber-Ep4 Dako, Denmark, 1:100 dilution), Ck17 (E3, Dako, Denmark, 1:50 dilution). Polyclonal rabbit anti-mouse immunoglobulins/HRP (Dako, Denmark, 1:100 dilution) were applied as enzyme-labeled secondary antibodies. 3.3-diaminobenzidine tetrahydrochloride (Sigma, USA) was used as a chromogen. Endogenous peroxidase was inactivated by incubation in $1 \% \mathrm{H}_{2} \mathrm{O}_{2}$ in phosphate buffer $(\mathrm{pH} 7.4)$ at the room temperature for 30 minutes. Appropriate positive and negative controls for ICH were included. The expression of antigens was considered positive in the case cytoplasm of thyrocytes intensive staining. As a result approximately 1000 thyrocytes were counted at high magnification $(\times 400)$ in each case.

\section{3. Statistical analysis}

Data were statistically processed using Statistica, version 11.0. When the p-value was $<0.05$, any differences were considered statistically significant. The Wilcoxon's non-parametric test was applied to compare the expression of TPO, Tg, Ck17 between different stages of the existence of SRIRM (when they accumulate RI (STAGE_1) and when they lose this ability (STAGE_2) in the same patients. The Mann-Whitney U-test was applied to compare the expression of TPO, Tg, Ck17 between PRIRM and RIAM. The chi-square test was used to compare frequencies of cytological signs (specific cellular phenotypes, structures, cystic degeneration) between PRIRM and RIAM and between different stages of the SRIRM.

\section{Results}

\section{1. Cytological features of PTC postoperative metastases}

A comparative cytologic study of thyrocytes' population of postoperative PTC metastases with different iodine accumulating ability was performed. From the point of view of cytology, when the metastases had the ability to accumulate RI (RIAM), they had the regular structure of cellular groups. These cellular groups were represented as homogeneous thyrocytes without signs of atypia (Fig. 1, a). In contrast, in a case of RI accumulation loss (in SRIRM and PRIRM) cellular groups with irregular structure appeared. They are represented as polymorphic cells.

Some cellular phenotype, which differed from other cells of the thyrocyte population with cytomorphological and ICH characteristics in $40 \%$ of STAGE_2 of SRIRM and $30 \%$ PRIRM was found. Their positive antibody reaction to cytokeratins 7 and 8 instantiates an epithelial nature. These thyrocytes don't contain Tg and do not react to antibodies against epCAM. This cellular phenotype looked lighter among the other epithelial cells and had clear-cut edges (Fig. 1, b). Statistically significant difference was observed in the presence of this specific cellular phenotype of thyrocytes between metastases of STAGE_1 and STAGE_2 of SRIRM.

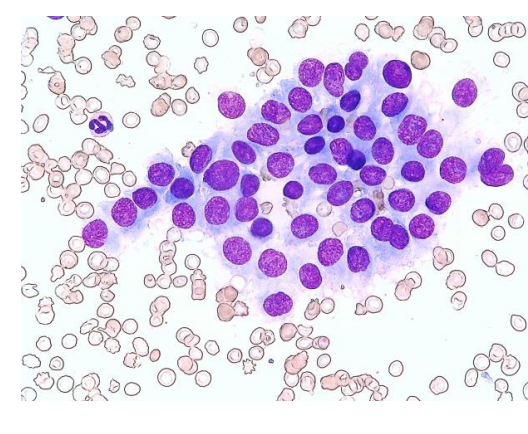

$a$

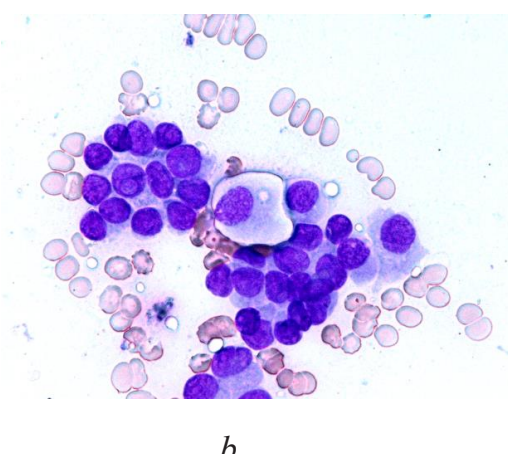

$b$

Fig. 1. The cellular groups in FNA smears of postoperative PTC metastases: $a$ - regular structure of cellular groups in RIAM; $b$ - particular cellular phenotype in STAGE_2 of SRIRM. $\times 400$ 
Besides, there are specific cellular complexes of psammous bodies and vacuolated cells in $20 \%$ of PRIRM and $46 \%$ of STAGE_ 2 of SRIRM. These complexes were absent in metastases, when they had an ability to accumulate RI. (Fig. 2).

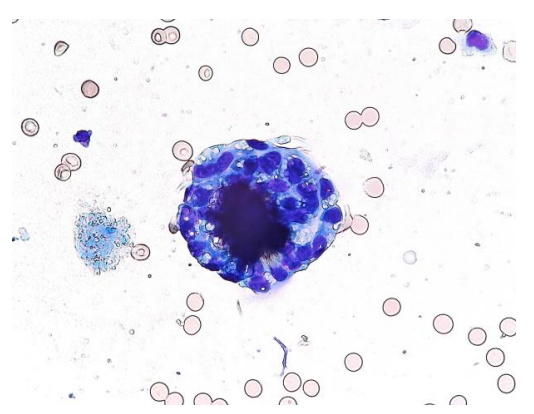

Fig. 2. Specific cellular complexes of psammous bodies and vacuolated cells in FNA smear of STAGE_2 of SRIRM. $\times 400$

Also, in $43 \%$ of PRIRM and $40 \%$ of cases of STAGE_ 2 of SRIRM there are cytological sign of cystic degeneration, in contrast to metastases, when they had an ability to accumulate RI. The data of the frequency of the studied cytological features in FNA smears of postoperative PTC metastases are given in Table $\mathbf{1 .}$

\section{Table 1}

The frequency of various cytological signs in FNA smears of postoperative PTC metastases (RIAM, PRIRM and SRIRM)

\begin{tabular}{|c|c|c|c|c|}
\hline \multirow[b]{2}{*}{ Cytological features } & \multirow{2}{*}{$\begin{array}{l}\text { RIAM } \\
(\mathbf{n}=\mathbf{2 5})\end{array}$} & \multirow{2}{*}{$\begin{array}{l}\text { PRIRM } \\
(\mathbf{n}=\mathbf{3 0})\end{array}$} & \multicolumn{2}{|c|}{ SRIRM (n=15) } \\
\hline & & & $\begin{array}{c}\text { Stage of RI accumulation } \\
\text { (STAGE_1) }\end{array}$ & $\begin{array}{c}\text { Stage of lost of RI accumulation } \\
\text { (STAGE_2) }\end{array}$ \\
\hline Special cellular phenotype & $0 \% \#$ & $30 \%(9)$ & $0 \% *$ & $40 \%(6)$ \\
\hline Cystic degeneration & $4 \%(1) \#$ & $43 \%(13)$ & $6.7 \%(1)^{*}$ & $40 \%(6)$ \\
\hline $\begin{array}{c}\text { Complexes of psammous } \\
\text { body and thyrocytes }\end{array}$ & $0 \% \#$ & $20 \%(6)$ & $0 \% *$ & $46.6 \%(7)$ \\
\hline
\end{tabular}

Note: RIAM - radioiodine-avid metastases, successfully treated using the radioiodine therapy, PRIRM - radioiodine-refractory metastases, which never accumulated radioiodine, SRIRM - which previously were responsive to radioiodine and eventually lose the ability to accumulate it; \#-p<0.05 in comparison RIAM with PRIRM; * $-p<0.05$ in comparison STAGE_1 with the STAGE_2 of SRIRM

So, conducted researches demonstrate appearance of the special cellular phenotype, complexes of psammous body and thyrocytes and cystic degeneration in FNA smears of primary radioiodine-refractory metastases and the stage of lost radioiodine accumulation (STAGE_2) of SRIRM, compared to radioiodine-avid metastases and the stage of radioiodine-accumulation (STAGE_1) of SRIRM.

\section{2. Immunocytochemical features of PTC postoperative metastases}

The statistically significant reduction of the expression of TPO and Tg at the level of significance $p<0.05$ between RIAM and PRIRM was shown ( $p=0$ and $p=0.000028$ respectively). The statistically significant reduction in the expression of TPO and Tg at the level of significance $p<0.05$ between different stages of the existence of SRIRM (STAGE_1 and STAGE_2) was shown ( $p=0.003346$ for both antigens) (Fig. 3). However, in the smears of RIAM (which were successfully treated by RI), the percentage of TPO-positive thyrocytes reached $100 \%$. Whereas, in the FNA smears of STAGE_1 of SRIRM the percentage of TPO-positive cells was $15-75 \%$ of thyrocytes. The difference between these groups of metastases in the expression of TPO was statisti- 
cally probable at the level of significance $p<0.05$ with the non-parametric Mann-Whitney U-test $(p=0,000047)$. At the same time, after the loss of RI accumulation ability (STAGE_2), the FNA smears of SRIRM had only 0-7\% of TPO-positive cells.

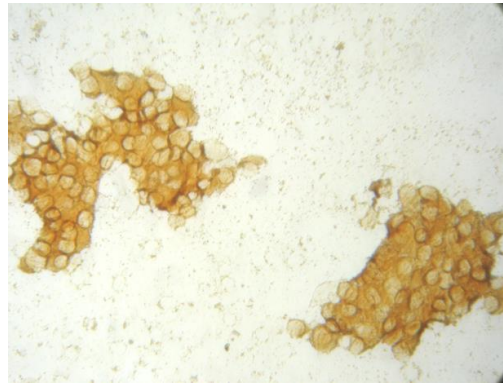

$a$

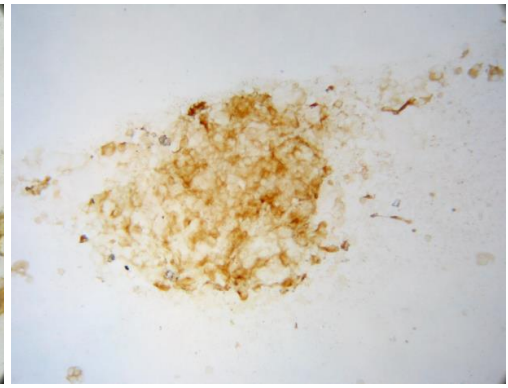

$b$

Fig. 3. Immunocytochemical reaction with antibody to thyroid peroxidase in FNA smears of postoperative PTC radioiodine accumulating metastases: $a$-intensive expression of thyroid peroxidase in punctate of RIAM; $b$ - mosaic expression of thyroid peroxidase in punctate of

STAGE_1 of SRIRM, $\times 200$

A statistically significant difference between STAGE_2 and STAGE_1 of SRIRM and as well as between PRIRM and RIAM was not shown with the $\mathrm{Ck} 17$-positive cells percentage at the level of significance $p<0.05(p=0.108810, p=0.910258)$.

The data of the frequency of the studied imunocytochemical features in FNA smears of postoperative PTC metastases are given in Table 2.

Table 2

The Median values $(M e, \%)$ of thyrocytes' percentage with antigens' expression (TPO, Tg, cK17) in FNA smears of postoperative PTC metastases (RIAM, PRIRM, SRIRM)

\begin{tabular}{ccccc}
\hline Imunocytochemacal features & $\begin{array}{c}\text { RIAM } \\
(\mathbf{n = 2 5})\end{array}$ & $\begin{array}{c}\text { PRIRM } \\
(\mathbf{n = 3 0})\end{array}$ & $\begin{array}{c}\text { Stage of RI accumulation } \\
\text { (STAGE_1) }\end{array}$ & $\begin{array}{c}\text { Stage of lost of RI } \\
\text { accumulation (STAGE_2) }\end{array}$ \\
\hline TPO-positive cells $(\mathrm{Me}, \%)$ & $77.5 \% \#$ & $0 \%$ & $20 \%^{*}$ & $0 \%$ \\
Tg-positive cells $(\mathrm{Me}, \%)$ & $92.5 \% \#$ & $56 \%$ & $90 \% \%^{*}$ & $50 \%$ \\
Ck17-positive cells $(\mathrm{Me}, \%)$ & $0 \%$ & $0 \%$ & $0 \% *$ & $10 \%$
\end{tabular}

Note: RIAM - radioiodine-avid metastases, successfully treated using the radioiodine therapy, PRIRM-radioiodine-refractory metastases, which never accumulate radioiodine, SRIRM - which previously were responsive to radioiodine and eventually lose the ability to accumulate it; \#-p<0.05 in comparison RIAM with RIRM; ${ }^{*}{ }_{-} p<0.05$ in comparison STAGE_ 1 with STAGE_2 of SRIRM

So, the statistically significant difference of the expression of thyroid peroxidase and thyroglobulin between radioiodine accumulating metastases and primary radioiodine-refractory metastases, and between different stages of the existence of secondary radioiodine-refractory metastases (STAGE_1 and STAGE_2) was shown. Moreover, the different efficacy of radioiodine-therapy depending on thyroid peroxidase-positive cells percentage in FNA smears of metastases was demonstrated.

\section{Discussion}

The study of FNA smears of postoperative PTC metastases with different iodine accumulating ability revealed cytological changes and changes of some antigens' expression that occurred in the thyrocyte' population in course of the loss of RI-accumulation ability. The 
conducted researches demonstrate the appearance of special cellular phenotypes and complexes in FNA smears of PRIRM and STAGE_2 of SRIRM, compared to RIAM and STAGE_1 of SRIRM. There is an emergence of some specific cellular phenotype that have no expression of Tg (marker of differentiated thyrocytes), which is relevant to the accumulation of RI during the loss of the ability to RI accumulation. The special cellular phenotype does not react with anti-epCAM (Ber EP4 antigen) antibodies. This is somewhat unexpected because epCAM, consisting of two glycoproteins, 34 and $39 \mathrm{kDa}$, is found on the cell surface and in the cytoplasm of virtually all epithelial cells, with very few exceptions. In particular, epCAM expression is typical of follicular (A-cells), Hurthle (B-cells) and parafollicular (C-cells) cells of the thyroid, both in health and cancer [14]. EpCAM - adhesion molecules of epithelial cells, they also considered to be one of the key factors in the process of epithelial-mesenchymal transition, when epithelial cells lose their polarity and intercellular adhesion and acquire the ability to migration. Probably, these characteristics of specific cellular phenotype in explored metastases are a source of RI refractoriness.

Also, there are specific cellular complexes of psammoma bodies and vacuolated thyrocytes in PRIRM and STAGE_2 of SRIRM that were absent in RI-accumulation metastases. Cytopathologists describe the numerous psammoma bodies in the single tissue fragment in a FNA smears from a diffuse sclerosing variant of PTC [15]. This variant of PTC is recognized as aggressive variants by the WHO. Probably, we can consider these complexes as a cytopathological sign of thyroid carcinoma' aggression and RI-refractoriness. However, this assumption requires further investigation.

The data regarding the tumors' resistance to the commonly used therapy has shown that the clonal tumor heterogeneity may be based on its development [16]. Previously there was a demonstration of the population heterogeneity in follicular epithelium only on the histological sections of the thyroid nodule's tissue [17]. We were the first to conduct the study of the tumor heterogeneity in the FNA smears of PTC metastases.

There is a statistically significant reduction of PRIRM, compared to RIAM and of STAGE_2, compared to STAGE_1 of SRIRM in the expression of TPO and Tg, which is momentous in the accumulation and retention of RI by cells of follicular epithelium. The received results do not contradict the literature data where researcher's ICH investigations of the RIRM showed a significant reduction in their tissue Tg, TPO, and their mRNA $[18,19]$. However, studies of these antigens' expression in FNA smears of metastases during the development of secondary radioiodine refractoriness were first conducted in this work. The appearance of special cellular phenotypes and complexes, the loss of antigens which take part in the accumulation and RI retention (TPO, Tg) may be a manifestation of the loss of cytological signs of high differentiation of thyrocytes that cause successful RI treatment. These data do not contradict the previously obtained results in the study of the histological material of PTC and their metastases [20].

TPO-antigen is related to the accumulation of RI [21,22]. We can see, that the median value of TPO-positive cells percentage for RIAM, successfully treated using the radioiodine therapy $(M e=80 \%)$, was statistically probably higher than for the group of STAGE_ 1 that eventually became STAGE_ 2 of SRIRM $(M e=20 \%)$. It demonstrated different efficacy of RI therapy, depending on TPO-positive cells percentage in FNA smears of metastases. We can see that the loss of the ability to RI accumulation of metastases happens despite the initial moderate ability during the development of secondary radioiodine refractoriness. Therefore, probably the low percentage of TPO-positive cells (up to $15 \%$ ), observable in FNA smears of STAGE_1 of SRIRM, may be not enough for the successful RI therapy. Basically, the reason of this phenomenon can be behind the phenotypic heterogeneity of tumors. Apparently, thyrocytes that have TPO and Tg are able to accumulate RI and eventually are destroyed by this radiopharmaceutical means. And the development of secondary radioiodine refractoriness in metastases happens thanks to thyrocytes that do not contain TPO and Tg and are not able to RI accumulation.

\section{Conclusions}

The postoperative secondary radioiodine-refractory metastases of papillary thyroid carcinomas, which initially accumulated radioiodine (STAGE_1), but eventually lost this ability 
(STAGE_2) were chosen as modeling for the study of cytological and immunocytochemical cells' changes that occur in the dynamics of development of radioiodine refractoriness.

The statistically significant reduction of the expression of thyroid peroxidase and thyroglobulin between radioiodine accumulating metastases and primary radioiodine-refractory metastases and between different stages of the existence of secondary radioiodine-refractory metastases (STAGE_1 and STAGE_2) was shown.

The conducted researches demonstrate the appearance of special cellular phenotypes and complexes in FNA smears of primary radioiodine-refractory metastases and the stage of lost radioiodine accumulation (STAGE_2) of secondary radioiodine-refractory metastases, compared to radioiodine-avid metastases, and the stage of radioiodine-accumulation (STAGE_1) of secondary radioiodine-refractory metastases.

The median value of thyroid peroxidase-positive cells percentage for radioiodine-avid metastases, successfully treated, using the radioiodine therapy, was statistically probably higher than for the group of the radioiodine-accumulative stage of postoperative metastases (STAGE_1), which eventually became secondary radioiodine-refractory metastases. It demonstrated different efficacy of the radioiodine-therapy, depending on thyroid peroxidase-positive cells percentage in FNA smears of metastases.

Revealing the phenotypic heterogeneity of thyrocytes (presence of specific cellular phenotypes, structures, cystic degeneration) in FNA smears and a low percentage of thyroid peroxidase and thyroglobulin-positive thyrocytes allows the development of the method of cytological prediction of the effectiveness of the radioiodine therapy of postoperative metastases of papillary thyroid cancer. Cytopathologists can use these results to provide their clinicians and patients with pertinent additional diagnostic information, which could significantly impact patient management and outcome.

\section{Acknowledgments}

The authors gratefully acknowledge histological confirmation, provided by the Laboratory of Pathology, Institute of Endocrinology and Metabolism (the head - Tatyana Ivanovna Bogdanova, Doctor of Bioligical Sciences) and Yuri Mikhailovich Bozhok, Doctor of Bioligical Sciences (the head of Laboratory of Cytology)

\section{References}

[1] Bogdanova, T. I., Zurnadzhy, L. Y., Nikiforov, Y. E., Leeman-Neill, R. J., Tronko, M. D., Chanock, S. et. al. (2015). Histopathological features of papillary thyroid carcinomas detected during four screening examinations of a Ukrainian-American cohort. British Journal of Cancer, 113 (11), 1556-1564. doi: https://doi.org/10.1038/bjc.2015.372

[2] Drozd, V. M., Branovan, I., Shiglik, N., Biko, J., Reiners, C. (2018). Thyroid Cancer Induction: Nitrates as Independent Risk Factors or Risk Modulators after Radiation Exposure, with a Focus on the Chernobyl Accident. European Thyroid Journal, 7 (2), 67-74. doi: https://doi.org/10.1159/000485971

[3] Markovina, S., Grigsby, P. W., Schwarz, J. K., DeWees, T., Moley, J. F., Siegel, B. A., Perkins, S. M. (2014). Treatment Approach, Surveillance, and Outcome of Well-Differentiated Thyroid Cancer in Childhood and Adolescence. Thyroid, 24 (7), 1121-1126. doi: https://doi.org/10.1089/thy.2013.0297

[4] Haugen, B. R., Alexander, E. K., Bible, K. C., Doherty, G. M., Mandel, S. J., Nikiforov, Y. E. et. al. (2016). 2015 American Thyroid Association Management Guidelines for Adult Patients with Thyroid Nodules and Differentiated Thyroid Cancer: The American Thyroid Association Guidelines Task Force on Thyroid Nodules and Differentiated Thyroid Cancer. Thyroid, 26 (1), 1-133. doi: https://doi.org/10.1089/thy.2015.0020

[5] Busaidy, N. L., Cabanillas, M. E. (2012). Differentiated Thyroid Cancer: Management of Patients with Radioiodine Nonresponsive Disease. Journal of Thyroid Research, 2012, 1-12. doi: https://oi.org/10.1155/2012/618985

[6] Pacini, F., Ito, Y., Luster, M., Pitoia, F., Robinson, B., Wirth, L. (2012). Radioactive iodine-refractory differentiated thyroid cancer: unmet needs and future directions. Expert Review of Endocrinology \& Metabolism, 7 (5), 541-554. doi: https://doi.org/ 10.1586/eem.12.36

[7] Worden, F. (2014). Treatment strategies for radioactive iodine-refractory differentiated thyroid cancer. Therapeutic Advances in Medical Oncology, 6 (6), 267-279. doi: https://doi.org/10.1177/1758834014548188

[8] Vaisman, F., Carvalho, D. P., Vaisman, M. (2015). A new appraisal of iodine refractory thyroid cancer. Endocrine-Related Cancer, 22 (6), R301-R310. doi: https://doi.org/10.1530/erc-15-0300 
[9] Deandreis, D., Al Ghuzlan, A., Leboulleux, S., Lacroix, L., Garsi, J. P., Talbot, M. et. al. (2010). Do histological, immunohistochemical, and metabolic (radioiodine and fluorodeoxyglucose uptakes) patterns of metastatic thyroid cancer correlate with patient outcome? Endocrine Related Cancer, 18 (1), 159-169. doi: https://doi.org/10.1677/erc-10-0233

[10] Rivera, M., Ghossein, R. A., Schoder, H., Gomez, D., Larson, S. M., Tuttle, R. M. (2008). Histopathologic characterization of radioactive iodine-refractory fluorodeoxyglucose-positron emission tomography-positive thyroid carcinoma. Cancer, 113 (1), 48-56. doi: https://doi.org/10.1002/cncr.23515

[11] Zelinskaya, A. V. (2019). Cytokeratin 17 and thyroid peroxidase as immunocytochemical markers for reoperative prediction of radioiodine resistance and the effectiveness of radioiodine therapy of papillary thyroid carcinoma. Oncology, 21 (1), 31-35.

[12] Pathology and Genetics of Tumours of Endocrine Organs (2004). WHO Classification of Tumours. IARC Press: Lyon, 320.

[13] Zelinskaya, A. (2019). Immunocytochemical characteristics of thyrocytes in radioiodine refractory metastases of papillary thyroid cancer. Experimental Oncology, 41 (4). doi: https://doi.org/10.32471/exp-oncology.2312-8852.vol-41-no-4.13705

[14] Latza, U., Niedobitek, G., Schwarting, R., Nekarda, H., Stein, H. (1990). Ber-EP4: new monoclonal antibody which distinguishes epithelia from mesothelial. Journal of Clinical Pathology, 43 (3), 213-219. doi: https://doi.org/10.1136/jcp.43.3.213

[15] Lastra, R. R., LiVolsi, V. A., Baloch, Z. W. (2014). Aggressive variants of follicular cell-derived thyroid carcinomas: A cytopathologist's perspective. Cancer Cytopathology, 122 (7), 484-503. doi: https://doi.org/10.1002/cncy.21417

[16] Almendro, V., Marusyk, A., Polyak, K. (2013). Cellular Heterogeneity and Molecular Evolution in Cancer. Annual Review of Pathology: Mechanisms of Disease, 8 (1), 277-302. doi: https://doi.org/10.1146/annurev-pathol-020712-163923

[17] Studer, H., Gerber, H., Zbaeren, J., Peter, H. J. (1992). Histomorphological and immunohistochemical evidence that human nodular goiters grow by episodic replication of multiple clusters of thyroid follicular cells. The Journal of Clinical Endocrinology \& Metabolism, 75 (4), 1151-1158. doi: https://doi.org/10.1210/jcem.75.4.1400886

[18] Wang, C., Zhang, X., Li, H., Li, X., Lin, Y. (2017). Quantitative thyroglobulin response to radioactive iodine treatment in predicting radioactive iodine-refractory thyroid cancer with pulmonary metastasis. PLOS ONE, 12 (7), e0179664. doi: https:// doi.org/10.1371/journal.pone.0179664

[19] Huang, M., Batra, R. K., Kogai, T., Lin, Y. Q., Hershman, J. M., Lichtenstein, A. et. al. (2001). Ectopic expression of the thyroperoxidase gene augments radioiodide uptake and retention mediated by the sodium iodide symporter in non-small cell lung cancer. Cancer Gene Therapy, 8 (8), 612-618. doi: https://doi.org/10.1038/sj.cgt.7700354

[20] Ricarte-Filho, J. C., Ryder, M., Chitale, D. A., Rivera, M., Heguy, A., Ladanyi, M. et. al. (2009). Mutational Profile of Advanced Primary and Metastatic Radioactive Iodine-Refractory Thyroid Cancers Reveals Distinct Pathogenetic Roles for BRAF, PIK3CA, and AKT1. Cancer Research, 69(11), 4885-4893. doi: https://doi.org/10.1158/0008-5472.can-09-0727

[21] Liu, Y. Y., Stokkel, M. P., Pereira, A. M., Corssmit, E. P., Morreau, H. A., Romijn, J. A., Smit, J. W. A. (2006). Bexarotene increases uptake of radioiodide in metastases of differentiated thyroid carcinoma. European Journal of Endocrinology, 154 (4), 525-531. doi: https://doi.org/10.1530/eje.1.02123

[22] Furuya, F., Shimura, H., Suzuki, H., Taki, K., Ohta, K., Haraguchi, K. et. al. (2004). Histone Deacetylase Inhibitors Restore Radioiodide Uptake and Retention in Poorly Differentiated and Anaplastic Thyroid Cancer Cells by Expression of the Sodium/Iodide Symporter Thyroperoxidase and Thyroglobulin. Endocrinology, 145 (6), 2865-2875. doi: https://oi.org/10.1210/ en.2003-1258 